\title{
SOME OCULAR COMPLICATIONS OF SCRUB TYPHUS*
}

\author{
BY \\ J. MACASKILL, R.A.M.C, \\ S.E.A.C.
}

THE spread of the war to tropical regions has directed attention to certain diseases. One of these is scrub typhus, and as many ophthalmologists in England are probably unfamiliar with the disease a short account of it is given, and mention is made of some of the ocular conditions I have found associated with it.

Scrub typhus (Tsutsugamushi Disease) is one of the large and numerous groups of typhus fevers, which also includes the epidemic louse-borne typhus of Eastern Europe.

The causal organism is a rickettsia, the rickettsia orienta which is believed to be transmitted to man by the larvae of a mite of the genus Trombiculae. The disease is endemic in rodents in the countries of Eastern Asia, and larval mites infesting such rodents become infected. In the adult stage the mite is vegetative in habits and so is not capable of transmitting the disease. However, the infection is passed hereditarily to the next generation of larvae, which may convey rickettsia to further rodents or man.

In South-East Asia such larval mites abound in many localised areas of scrub jungle. Men, while walking, crawling or lying in such areas are liable to be infected, since the larval mite attaches itself to the skin, burrows into the superficial layers, and so passes the rickettsia into the body.

Clinical features.-There is an incubation period of about twelve days. The site of entry may be marked by an eschar; and fever, severe toxaemia, headache and prostration ensue. In some, the patients become unconscious, in others mentally disoriented: Diffuse glandular enlargement and a maculo-papular rash are common. The severity of the disease varies in different regions; the mortality rate being 10 per cent. to 15 per cent. in most outbreaks. The diagnosis is assisted by the Weil-Felix reaction, which becomes positive about the tenth day of the illness. In this reaction the serum of an infected patient agglutinates an " $O$ " suspension of Proteus XX.

General pathology.-Together with the severe toxaemia already referred to, the essential pathological change is a generalised vasculitis affecting the arterioles and smaller vessels. This results in necrosis and destruction of the vessel walls, with extravasation of blood into surrounding tissues, and vascular blockage either by thrombosis or cellular infiltrations. These are composed of proliferated vascular endothelium and small irregular cuffs of histiocytes and plasma cells. The lesions are patchily distributed

* Received for publication, August 8, 1945. 
throughout all organs and the central nervous system. Generalised congestion of the brain and meninges is commonly seen at postmortem, together with haemorrhages and vascular changes.

Some ocular complications. - The conditions of severe toxaemia and generalised vasculitis gives rise to certain ocular changes, some due to the toxaemia, others to areas of vasculitis occurring in the eye or visual pathway. Seventy cases which occurred in Assam were examined, and notes are given of those showing ocular changes. The Weil-Felix test was positive in all, except one who died too early in the disease for the test to be significant.

Congestion of the conjunctiva occurs invariably in the acute phase of the disease, so constantly that it is a clinical feature rather than a complication of the disease.

Subjunctival haemorrhages were seen in four cases. They are usually of small extent but one case showed extensive bilateral haemorrhages. It is interesting to note that similar haemorrhages are met with in malaria, a point to be kept in mind in diagnosis, since both diseases occur in similar regions.

Marked engorgement of the retinal veins was noted in seven cases. This probably represents an under-estimate of the frequency with which some degree of engorgement is present, as only marked changes in calibre were considered.

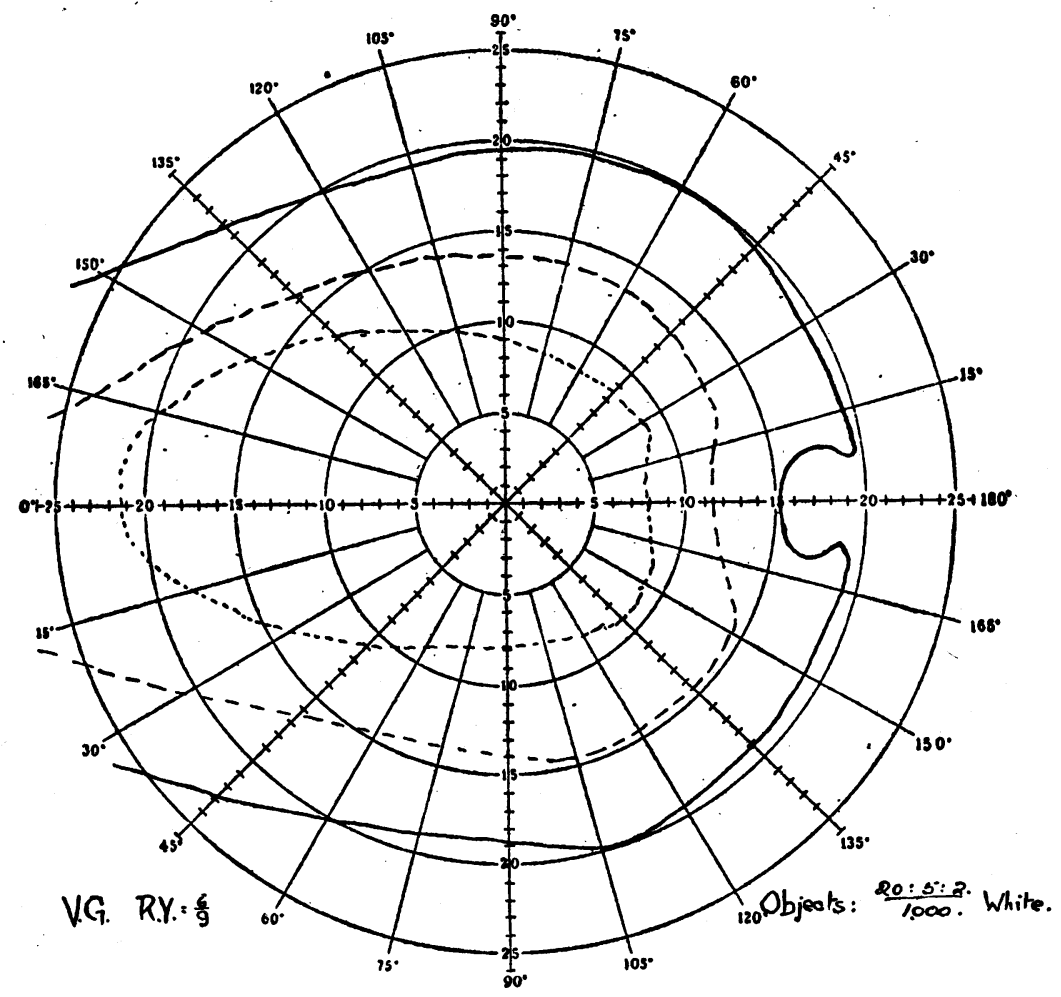




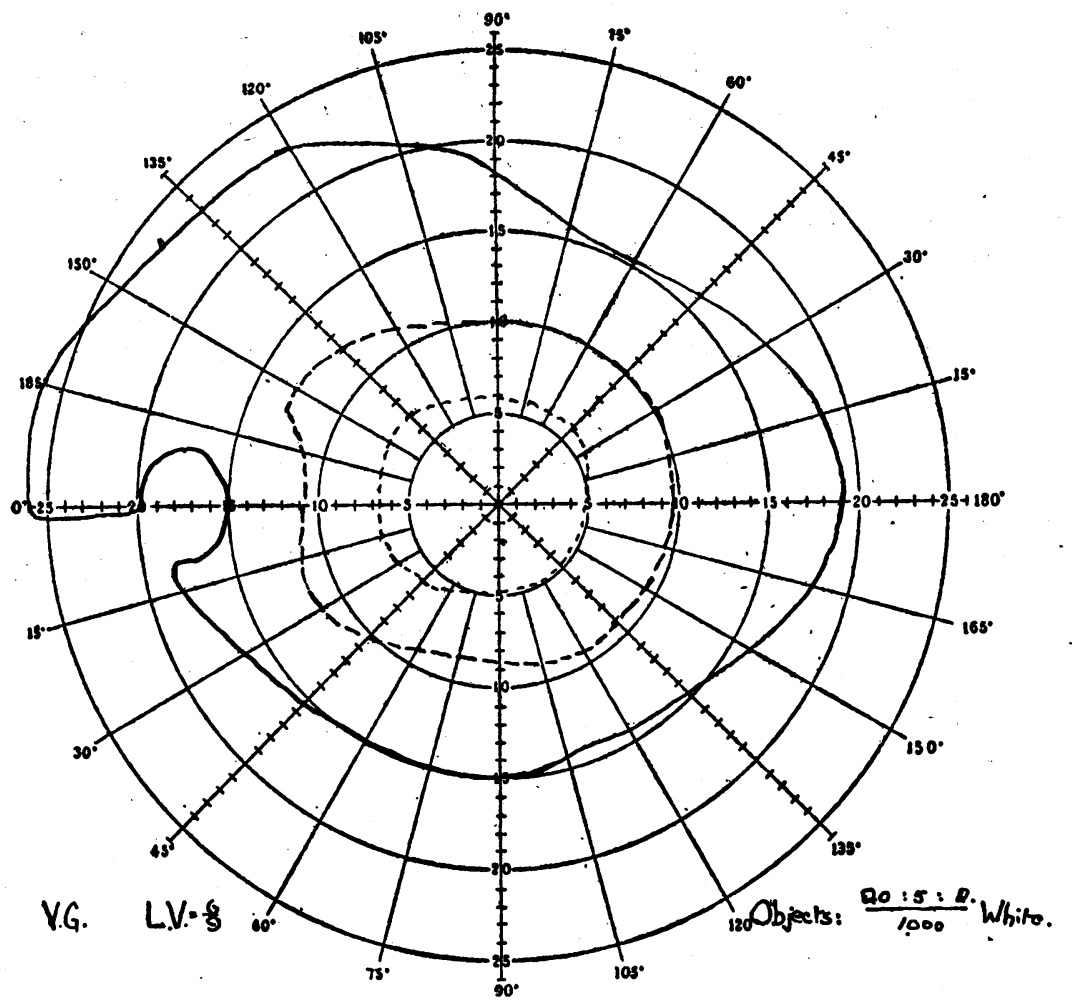

Papilloedema was present in four cases and was bilateral in all except one. In three of them there was increase of intracranial pressure and this was associated with increase in the protein content, and cells were increased to 60 or so per c.mm.

Retinal haemorrhages occurred in four cases, two of whom also had papilloedema. They were usually small, few in number and in the nerve fibre layer, being situated in the region immediately surrounding the optic disc. One case, however, showed numerous haemorrhages which encircled the discs on both sides.

One interesting case of bilateral optic atrophy was seen six months after the acute stage of the illness; and a summary of the case and the central field charts are given.

\section{Case of L./Cpl. V. G., aged 30 years}

The patient was acutely ill for two weeks, and one week later noticed a defect in the vision of both eyes. After three days he could only see hand movements close up to the face, and this visual state remained unchanged for six weeks. Thereafter vision commenced to improve, and at the time of examination he could see quite well straight ahead, but had difficulty in seeing at the sides and at night. No quinine or arsenicals had been given. 
When examined the vision was $6 / 9$ in both eyes and there was bilateral optic atrophy. The Weil-Felix reaction was positive, and the Khan test negative. There was great depression of the periphery of the field of vision and the nature of the remaining central field will be seen. The shélving nature of the edge of the visual area is interesting, particularly as no change had occurred when the fields were examined two months later.

\section{Discussion}

The haemorrhages found in the subconjunctiva and retina probably arose from minute areas of vasculitis with damage to vessel walls and consequent leakage of blood. In the formation of the retinal haemorrhages some degree of back pressure was probably also a factor, for marked venous engorgement was found in some and many had some degree of back pressure on the retinal veins. The cause of this is doubtless the cerebral and meningeal congestion known to occur, and the papilloedema found in some can be similarly explained.

The optic atrophy and the associated loss of the visual periphery is interesting and its cause difficult to determine. The rapid and fairly complete initial loss, and the extent of recovery favours a toxic origin. The shelving nature of the edge of the seeing area suggested that some further recovery would have taken place, but, as remarked, no change was noted after an interval of two months.

In considering the optic atrophy, it is interesting that a considerable number of cases of scrub typhus develop a nerve deafness. It is odd that this nerve should be singled out, and, as in the case of the optic nerve, the cause may be a toxic one.

I have pleasure in thanking Major M. T. Parker, R.A.M.C., for his help in the pathological aspects of this disease, and make acknowledgment to the appropriate Army authorities for permission to publish these notes.

\section{ANNOTATION}

\section{Word blindness}

The paper published in our last issue on word blindness is of great interest, and the suggestions as to teaching a backward child to read most valuable. All of us must have met with similar cases either at school or in after life. It is of course conceivable that the school boy, who dreads being put on to construe in class, may not have prepared his lesson beforehand, either through sheer laziness or from having been employed in some other more interesting occupation. In our own school days we were something of an authority on ex tempore improvisation in translating a Latin sentence, 